\title{
Changes of Exercise, Screen Time, Fast Food Consumption, Alcohol, and Cigarette Smoking during the COVID-19 Pandemic among Adults in the United States
}

\author{
Liwei Chen ${ }^{1, *}$, Jian Li ${ }^{2,3}{ }^{\oplus}$, Tong Xia ${ }^{1}$, Timothy A. Matthews ${ }^{2}$, Tung-Sung Tseng ${ }^{4} \oplus$, Lu Shi ${ }^{5}$, Donglan Zhang ${ }^{6}$, \\ Zhuo Chen ${ }^{6,7}{ }^{\mathbb{D}}$, Xuesong Han ${ }^{8}$, Yan $\mathrm{Li}^{9}{ }^{9}$, , Hongmei $\mathrm{Li}^{10}$, Ming Wen ${ }^{11}{ }^{1}$ and Dejun Su ${ }^{12}$
}

1 Department of Epidemiology, Fielding School of Public Health, University of California Los Angeles, Los Angeles, CA 90095, USA; xiatong@g.ucla.edu

2 Department of Environmental Health Sciences, Fielding School of Public Health, University of California Los Angeles, Los Angeles, CA 90095, USA; jianli2019@ucla.edu (J.L.); tmatthews@ucla.edu (T.A.M.)

3 School of Nursing, University of California Los Angeles, Los Angeles, CA 90095, USA

4 Department of Behavioral and Community Health Sciences, LSUHSC School of Public Health, 2020 Gravier Street, Room 213, New Orleans, LA 70112, USA; ttseng@lsuhsc.edu

5 Department of Public Health Sciences, Clemson University, Clemson, SC 29634, USA; lus@clemson.edu

6 Department of Health Policy and Management, College of Public Health, University of Georgia, Athens, GA 30602, USA; dzhang@uga.edu (D.Z.); zchen1@uga.edu (Z.C.)

7 School of Economics, Faculty of Humanities and Social Science, University of Nottingham Ningbo China, Ningbo 315100, China

check for

updates

Citation: Chen, L.; Li, J.; Xia, T.; Matthews, T.A.; Tseng, T.-S.; Shi, L.; Zhang, D.; Chen, Z.; Han, X.; Li, Y.; et al. Changes of Exercise, Screen Time, Fast Food Consumption, Alcohol, and Cigarette Smoking during the COVID-19 Pandemic among Adults in the United States. Nutrients 2021, 13, 3359. https:// doi.org/10.3390/nu13103359

Academic Editor: Jose Lara

Received: 19 August 2021

Accepted: 21 September 2021

Published: 25 September 2021

Publisher's Note: MDPI stays neutral with regard to jurisdictional claims in published maps and institutional affiliations.

Copyright: (c) 2021 by the authors. Licensee MDPI, Basel, Switzerland. This article is an open access article distributed under the terms and conditions of the Creative Commons Attribution (CC BY) license (https:// creativecommons.org/licenses/by/ $4.0 /)$.
8 Surveillance and Health Services Research Program, American Cancer Society, Atlanta, GA 30303, USA; xuesong.han@cancer.org

9 Department of Population Health Science and Policy, Department of Obstetrics, Gynecology, and Reproductive Science, Icahn School of Medicine at Mount Sinai, New York, NY 10029, USA; yan.li1@mountsinai.org

10 Department of Media, Journalism and Film, Miami University, Oxford, OH 45056, USA; 1ih19@miamioh.edu

11 Department of Sociology, University of Utah, Salt Lake City, UT 84112, USA; ming.wen@soc.utah.edu

12 Department of Health Promotion, College of Public Health, University of Nebraska Medical Center, Omaha, NE 68198, USA; dejun.su@unmc.edu

* Correspondence: cliwei86@ucla.edu

Abstract: Objective: To investigate the impact of the COVID-19 pandemic on multiple lifestyle changes among adults in the United States (USA). Methods: We conducted a survey, the Health, Ethnicity, and Pandemic (HEAP) Study, in October 2020 among USA adults. Participants were selected from the United States using 48 sampling strata, including age, race, ethnicity, education, and gender, and were asked to report five lifestyle behaviors (i.e., exercise time, screen time, fastfood meal consumption, alcohol drinking, and cigarette smoking) before and during the COVID-19 pandemic. The associations of sociodemographic factors with each lifestyle change were estimated using weighted multivariable logistic regression models. Results: All 2709 HEAP participants were included in this study. Compared to pre-pandemic, the time spent on exercise decreased ( 32.06 vs. $38.65 \mathrm{~min} /$ day; $p<0.001)$ and screen time increased ( 6.79 vs. $5.06 \mathrm{~h} /$ day; $p<0.001)$ during the pandemic. The percentage of individuals who reported consuming fast-food meals $\geq 3$ times/week decreased from $37.7 \%$ before the pandemic to $33.3 \%$ during the pandemic. The percentage of heavy drinkers ( $\geq 5$ times /week) increased from $20.9 \%$ before the pandemic to $25.7 \%$ during the pandemic. Among smokers, heavy smoking ( $\geq 11$ cigarettes/day) increased from $5.8 \%$ before the pandemic to $7.9 \%$ during the pandemic. We also identified subgroups who were more vulnerable to adverse influences from the pandemic, including racial/ethnic minority groups and young adults. Conclusions: The COVID-19 pandemic had negative impacts on multiple lifestyle behaviors among Americans. Mitigating such negative impacts of COVID-19 requires effective interventions, particularly for some vulnerable subgroups.

Keywords: COVID-19; fast food; exercise; screen time; alcohol; smoking; lifestyles 


\section{Introduction}

The novel coronavirus disease (COVID-19), induced by severe acute respiratory syndrome coronavirus 2 (SARS-CoV-2), has caused a worldwide pandemic since March 2020. SARS-CoV-2 is a single-stranded RNA virus and the third coronavirus ((i.e., since SARS 2003, Middle East Respiratory Syndrome (MERS) 2012)) that has caused severe diseases in human in the past two decades [1]. COVID-19 is known to spread primarily via respiratory droplets during close contact. The symptoms of COVID-19 mainly appear 2-14 days after exposure to the virus and can be ranged from mild to severe, including fatigue, headache, fever, dry cough, sore throat, new loss of taste and smell, muscle or body aches, diarrhea, difficulty breathing, sepsis, and acute respiratory failure [2]. Approximately $5 \%$ of patients who were infected with COVID-19 had severe symptoms and needed intensive care [3]. As of 6 September 2021, more than 220 million people have been infected by the SARS-CoV-2 and 4.5 million died worldwide [4].

In the 2003 Severe Acute Respiratory Syndrome (SARS) pandemic, several studies documented changes of health behaviors in people living in the most infected areas, such as China [5,6]. During the current COVID-19 pandemic, many countries, including the United States (USA), implemented measures and recommendations to restrict non-essential activities and reduce the spread of the disease. While these restrictions were helpful to reduce human-to-human infection of COVID-19, they could result in profound changes in people's normal daily activities and behaviors, including travel, shopping, exercise, food consumption, and eating patterns, as well as smoking and alcohol drinking. For example, levels of daily physical activity and exercise might be significantly reduced due to the closures of gyms, recreational facilities, group exercise classes, and travel restrictions. On the other hand, working, meeting, and learning remotely are likely to increase screen time and sedentary behaviors. In addition, restaurant closures and increased online grocery shopping might also change food consumption and dietary behaviors.

Since the beginning of the COVID-19 pandemic, several studies from different countries have observed undesired lifestyle changes among their populations, such as less exercise, more sedentary behavior, unhealthy dietary patterns, and increased alcohol consumption and cigarette smoking in Canada [7], Italy [8,9], Brazil [10], and Poland [11], as well as an international study via online survey [12]. To the best of our knowledge, only one small-scale local study examined physical activity and diet during the pandemic using data from 112 desk workers in the USA [13]. Two other USA studies only targeted alcohol drinking or cigarette smoking $[14,15]$.

The objective of our study was to investigate the impact of COVID-19 on multiple lifestyle behaviors in USA adults and to identify sub-groups who were more adversely impacted by the COVID-19 pandemic, using a nationally representative, population-based sample.

\section{Methods}

\subsection{Study Design and Population}

This analysis was conducted using the data from the Health, Ethnicity, and Pandemic (HEAP) Study. HEAP is survey designed by a consortium of investigators from several universities in the USA, led by the Center for Reducing Health Disparities at the University of Nebraska Medical Center. The HEAP survey was carried out by the National Opinion Research Center (NORC) at the University of Chicago with the sample randomly drawn from NORC's AmeriSpeak Panel and Dynata panel. AmeriSpeak is a probability-based mixed-mode panel designed to be representative of the USA non-institutionalized population using a multi-stage address-based sample design. Participants were selected using 48 sampling strata, including age, race, education, and gender following the American Association for Public Opinion Research guidelines. Detailed information regarding the panel recruitment and selection methodology can be found in the previous publication [16] Figure 1 provides the flowchart of participant selection in the AmeriSpeak panel. The AmeriSpeak panel was supplemented with respondents who were Asian-American from 
the Dynata panel. The HEAP survey was conducted between October and November 2020 and was delivered both in English and Spanish through the internet or telephone. Participants received $\$ 3$ in cash or the equivalent as an incentive to complete the survey. NORC implemented stratified sampling and poststratification weighting procedures to ensure the study sample was nationally representative of USA adults (age $\geq 18$ years). The study was approved by the Institutional Review Board at the NORC.

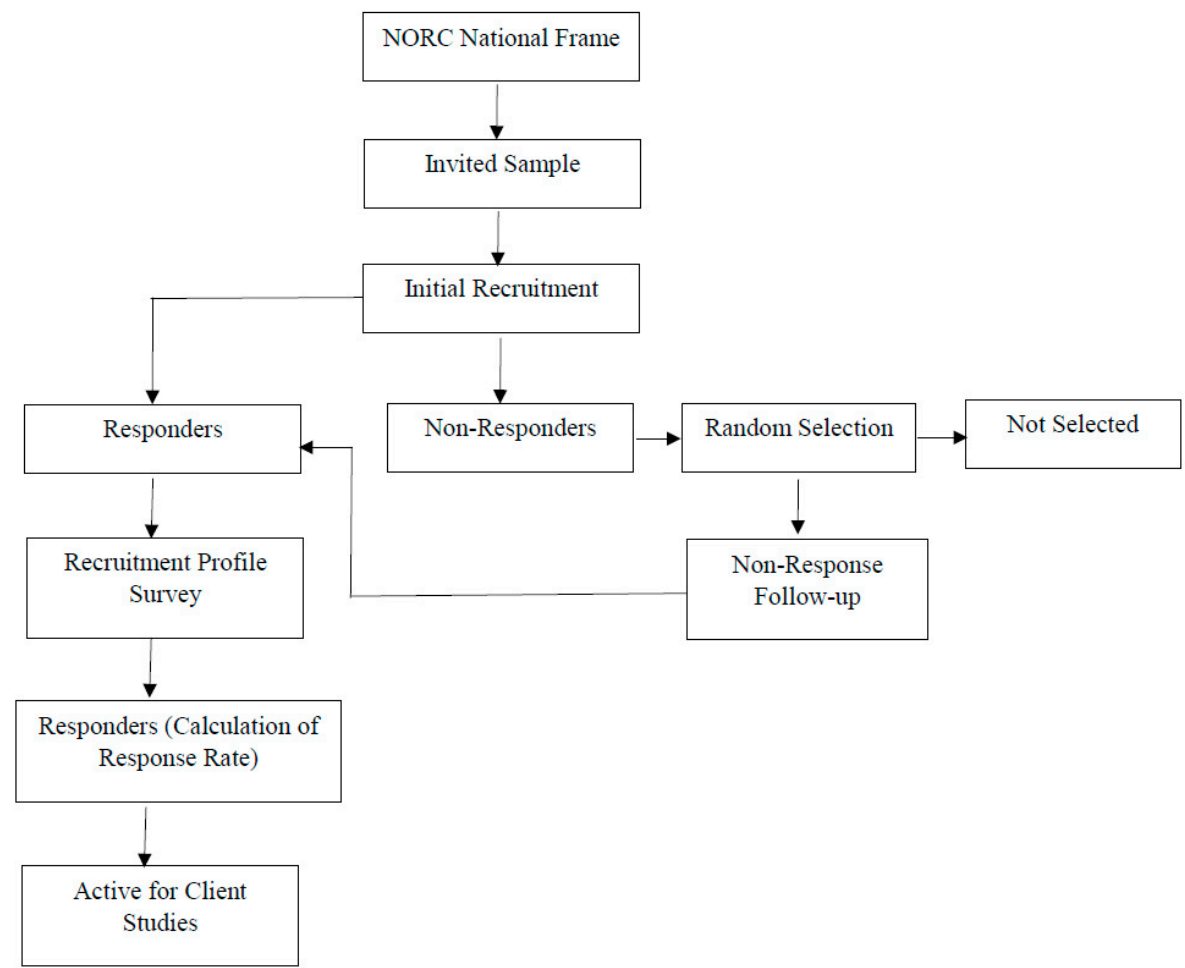

Figure 1. AmeriSpeak Panel Recruitment Methodology-Participants Selection Flowchart: The NORC national frame is an area probability sample of 3 million households based on the USA Postal Service Sequence File (covers approximately $97 \%$ of USA households) [16]. Abbreviations: NORC: National Opinion Research Center.

\subsection{Survey Design and Measures}

The HEAP is a cross-sectional study in design and the survey included questions related to mental health, lifestyle changes, racial discrimination, financial status, and healthcare utilization. Lifestyle changes were assessed using five questions asking participants to report the following behaviors before and during the pandemic: (1) number of minutes spent on exercise (e.g., running, walking, swimming, sports, yoga, and strength training) per day; (2) the number of fast-food meals (e.g., burgers, fries, and pizza) consumed per week; (3) the number of hours spent on screen time (e.g., TV, computer, cellphone, iPad, etc.) per day; (4) the number of cigarettes smoked per day; and (5) the number of alcoholic drinks consumed per week (one drink is equivalent to a 12-ounce beer, a 5-ounce glass of wine, or a drink with one shot of liquor). The survey also collected sociodemographic variables such as age, sex, race/ethnicity, educational attainment, marital status, annual household income, and health insurance coverage.

\subsection{Statistical Analysis}

All statistical analyses were performed with poststratification weighting to account for the complex survey design and sampling procedures. Each lifestyle behavior, before or during the pandemic, was reported as weighted mean (standard error, SE) for continuous variables or weighted percentage (actual frequency) for categorical variables. Differences in these behaviors during the pandemic were compared to behaviors before the pandemic 
using the Wilcoxon Signed Rank Test for continuous variables and Bhapkar's Test for categorical variables. The prevalence of missing data ranged from 3.1 to $3.8 \%$ (including the response categories "Don't know" and "Prefer not to answer") for measures of lifestyle/behaviors. Changes in lifestyle behaviors were calculated (during pandemicbefore pandemic) and categorized as "desired change" or "undesired change" for each behavior. The "undesired change" category refers to decreased exercise, increased screen time, increased numbers of fast-food meals, increased cigarettes smoking, or increased alcohol drinking during the pandemic as compared with before the pandemic, respectively, for each behavior. Associations of sociodemographic factors with changes in lifestyle behaviors were estimated using logistic regression models. Sociodemographic factors included as independent variables in the logistic regression models were age (18-29, 30-44, 45-59, vs. $\geq 60$ years), sex (female vs. male), race/ethnicity (Non-Hispanic Blacks, Hispanics, Asians and Pacific Islanders (APIs), or Alaska Natives or Others vs. Non-Hispanic Whites), educational attainment (associate's degree, bachelor's degree, or higher vs. high school or less), marital status (widowed/divorced/separated or never married vs. married/living with a partner), annual household income $(\$ 25,000-49,999$ or $\geq \$ 50,000$ vs. $<\$ 25,000)$, and health insurance before the pandemic (uninsured, Medicare, or Medicaid vs. private insurance). All statistical analyses were performed with SAS statistical software (Version 9.4 SAS Institute Inc, Cary, NC, USA).

\section{Results}

\subsection{Characteristics of Study Participants}

All 2709 HEAP participants were included in this study. Among them, 20.5\% were aged between $18-29$ years, $25.5 \%$ between $30-44$ years, $24.2 \%$ between $45-59$ years, and $29.8 \% \geq 60$ years. The study population reflected the USA racial/ethnic composition, and the majority were Non-Hispanic Whites (61.3\%), followed by Hispanics (16.7\%), NonHispanic Blacks (11.9\%), and Asian and Pacific Islanders (6.4\%). Of all participants, 51.7\% were female, $34.3 \%$ had a bachelor's degree or higher, $58.2 \%$ were married or living with a partner, $58.6 \%$ had an annual household income $\geq \$ 50,000$, and $52.7 \%$ had private health insurance (Table 1).

Table 1. Sociodemographic characteristics of HEAP study participants.

\begin{tabular}{cc}
\hline Characteristics & $\% \mathbf{~ ( N )}$ \\
\hline Age groups & \\
$18-29$ years & $20.5(568)$ \\
$30-44$ years & $25.5(880)$ \\
$45-59$ years & $24.2(573)$ \\
Z60 years & $29.8(688)$ \\
Female & $51.7(1413)$ \\
Race/ethnicity & \\
Non-Hispanic White & $61.3(514)$ \\
Non-Hispanic Black & $11.9(590)$ \\
Asian and Pacific Islander & $6.4(1012)$ \\
Hispanic & $16.7(532)$ \\
American Indian or others & $3.6(58)$ \\
Education & \\
High school or less & $38.0(583)$ \\
Associates & $27.7(1073)$ \\
Bachelor's or higher & $34.3(1053)$ \\
Marital status & \\
Married/living with a partner & $58.2(1444)$ \\
Widowed/divorced/separated & $17.0(404)$ \\
Never married & $24.8(861)$ \\
Annual household income & \\
\hline
\end{tabular}


Table 1. Cont.

\begin{tabular}{cc}
\hline Characteristics & $\% \mathbf{~ ( N )}$ \\
\hline$<\$ 25,000$ & $18.8(562)$ \\
$\$ 25,000-\$ 49,999$ & $22.5(625)$ \\
$\geq \$ 50,000$ & $58.6(1522)$ \\
Health insurance before the pandemic & \\
Private & $52.7(1480)$ \\
Uninsured & $8.3(222)$ \\
Medicare & $21.3(535)$ \\
Medicaid & $17.7(452)$ \\
\hline
\end{tabular}

Data were presented as weighted percentage, \% (actual frequency, N).

\subsection{Lifestyle Behaviors before and during the COVID-19 Pandemic}

Table 2 describes the distributions of five lifestyle behaviors before and during the COVID-19 pandemic. Compared with the pre-pandemic level, the daily average time spent on exercise decreased (32.06 vs. $38.65 \mathrm{~min} /$ day; $p<0.001$ ), and screen time increased ( 6.79 vs. $5.06 \mathrm{~h} /$ day; $p<0.001)$ during the pandemic. Before the pandemic, $15.3 \%$ of individuals reported zero exercise time, and this proportion increased to $20.9 \%$ during the pandemic. The proportion of individuals who exercised $\geq 30 \mathrm{~min}$ per day also decreased from $56.4 \%$ before the pandemic to $45.4 \%$ during the pandemic. Individuals with screen time $\geq 4 \mathrm{~h}$ per day also increased from $59.5 \%$ pre-pandemic to $79.8 \%$ during the pandemic. For fast-food meals, the average consumption level was 1.41 times/week before the pandemic and decreased to 0.96 time/week during the pandemic. The percentage of zero consumption increased from $15.0 \%$ before the pandemic to $25.0 \%$ during the pandemic, and the percentage of consuming three or more fast-food meals per week decreased from $37.7 \%$ before the pandemic to $33.3 \%$ during the pandemic. The average alcohol drinking level among drinkers increased from 3.01 drinks / week before the pandemic to 4.24 drinks / week during the pandemic $(p<0.001)$. Non-alcoholic beverage drinking was unchanged during the pandemic ( $50.6 \%$ vs. $49.8 \%$ ), but the percentage of heavy drinkers ( $\geq 5$ drinks /week) increased from $20.9 \%$ before the pandemic to $25.7 \%$ during the pandemic. The average cigarette smoking level among smokers increased slightly from 9.29 times/week before the pandemic to 9.80 times/week during the pandemic $(p<0.001)$. The percentage of nonsmokers was similar before and during the pandemic ( 82.9 vs. $82.4 \%$ ). Quitting behavior was not statistically significant before and during the pandemic (only $0.2 \%$ of smokers became non-smokers). However, among smokers, heavy smoking ( $\geq 11$ cigarettes/day) increased from $5.8 \%$ pre-pandemic to $7.9 \%$ during the pandemic.

Figure 2 shows the percentage of participants who had undesired changes of five lifestyle behaviors (i.e., decreased exercise, increased screen time, increased numbers of fast-food meals, increased cigarettes smoking, or increased alcohol drinking during the pandemic as compared with before the pandemic). Overall, $31.2 \%$ of the participants experienced decreased exercise time, $60.4 \%$ increased screen time, $22.6 \%$ increased consumption of fast-food meals, $23.3 \%$ increased alcohol drinking, and $9.0 \%$ increased cigarette smoking during the pandemic compared to before the pandemic. 
Table 2. Changes of lifestyle behaviors between before and during the COVID-19 pandemic.

\begin{tabular}{|c|c|c|c|}
\hline Variables & $\begin{array}{l}\text { Before the } \\
\text { Pandemic }\end{array}$ & $\begin{array}{l}\text { During the } \\
\text { Pandemic }\end{array}$ & $p$ Values \\
\hline Total exercise time (min/day), mean (SE) & $38.65(2.02)$ & $32.06(1.66)$ & $<0.001$ \\
\hline Exercise time categories, \% (N) & & & $<0.001$ \\
\hline $0 \mathrm{~min} /$ day & $15.3(379)$ & $20.9(508)$ & \\
\hline 1-30 $\mathrm{min} /$ day & $28.3(722)$ & $33.7(888)$ & \\
\hline$\geq 30 \mathrm{~min} /$ day & $56.4(1517)$ & $45.4(1222)$ & \\
\hline Total screen time (hours/day), mean (SE) & $5.06(0.13)$ & $6.79(0.14)$ & $<0.001$ \\
\hline Screen time categories, \% (N) & & & $<0.001$ \\
\hline $0 \mathrm{~h} /$ day & $2.1(52)$ & $1.6(47)$ & \\
\hline$<0$ and $<4 \mathrm{~h} /$ day & $38.4(988)$ & $18.7(474)$ & \\
\hline$\geq 4 \mathrm{~h} /$ day & $59.5(1576)$ & $79.8(2095)$ & \\
\hline Fast-food meals intake (time/week), median (IQR) & $1.41(2.55)$ & $0.96(2.74)$ & $<0.001$ \\
\hline Fast-food meals intake categories, $\%(\mathrm{~N})$ & & & $<0.001$ \\
\hline 0 time/week & $15.0(400)$ & $25.0(663)$ & \\
\hline 1-2 time/week & $47.3(1142)$ & $41.7(1036)$ & \\
\hline$\geq 3$ times/week & $37.7(1061)$ & $33.3(904)$ & \\
\hline $\begin{array}{c}\text { Alcohol drinking (drinks/week) among drinkers, } \\
\text { median (IQR) }\end{array}$ & $3.01(5.13)$ & $4.24(6.92)$ & $<0.001$ \\
\hline Alcohol drinking categories, \% (N) & & & $<0.001$ \\
\hline 0 drink/week & $50.6(1409)$ & $49.8(1416)$ & \\
\hline 1-4 drinks/week & $28.5(806)$ & $24.6(687)$ & \\
\hline$\geq 5$ drinks/week & $20.9(395)$ & $25.7(507)$ & \\
\hline $\begin{array}{c}\text { Cigarette smoking (times/day) among smokers, } \\
\text { median (IQR) }\end{array}$ & $9.29(10.32)$ & $9.80(12.93)$ & $<0.001$ \\
\hline Cigarette smoking categories, \% (N) & & & $<0.001$ \\
\hline Non-smokers & $82.9(2190)$ & $82.4(2185)$ & \\
\hline $1-5$ cigarettes / day & $5.8(181)$ & $3.9(135)$ & \\
\hline $6-10$ cigarettes/day & $5.4(143)$ & $5.8(149)$ & \\
\hline$\geq 11$ cigarettes/day & $5.8(99)$ & $7.9(144)$ & \\
\hline
\end{tabular}

Data were presented as weighted mean (standard errors, SE) for continuous variables with normal distributions and median ((interquater range (IQR)) for continuous variables with non-normal distribution, and weighted percentage, \% (actual frequency, $\mathrm{N}$ ) for categorical variables. Before and during pandemic lifestyle difference were compared using Wilcoxon signed-rank test for continuous variables and Bhapkar's test for categorical variables.



Figure 2. Bar chart for percentage (\%) of undesired changes in 5 lifestyle behaviors during the COVID-19 pandemic. Changes in lifestyle behaviors were calculated (during pandemic-before pandemic) and categorized as "desired change" or "undesired change" for each behavior. The "undesired change" category refers to decreased exercise, increased screen time, increased numbers of fast-food meals, increased cigarettes smoking, or increased alcohol drinking during the pandemic as compared with before the pandemic, respectively, for each behavior. 


\subsection{Sociodemographic Factors Associated with Changes in Individual Lifestyle Behaviors}

The relationship between sociodemographic factors and undesired changes in the five lifestyle behaviors are presented in Table 3. Compared to old adults aged 60 years or over, younger adults aged between 18-29 and 30-44 had higher odds of increasing consumption of fast-food meals and alcohol drinking during the pandemic; the age groups of 30-44 years $(\mathrm{OR}=4.74 ; 95 \%$ CI: $1.80-12.48)$ and $45-59(\mathrm{OR}=3.37 ; 95 \% \mathrm{CI}: 1.28-8.85)$ years had higher odds of increasing smoking, and the age group of 30-44 years had higher odds (OR $=1.77$; $95 \%$ CI: 1.13-2.77) of reduction in exercise time. Women had higher odds (OR $=1.72 ; 95 \%$ CI: 1.29-2.30) of increasing screen time than men. Compared to Non-Hispanic Whites, NonHispanic Blacks (OR = 1.64; 95\% CI: 1.17-2.28) and Hispanics (OR = 2.30; 95\% CI: 1.64-3.24) had higher odds of decreasing exercise time; Hispanics had higher odds of increasing screen time (OR = 1.92; 95\% CI: 1.35-2.72); Non-Hispanic Blacks (OR = 1.49; 95\% CI: 1.03-2.17), Hispanics (OR $=1.75 ; 95 \% \mathrm{CI}: 1.21-2.52)$, and American Indian or others $(\mathrm{OR}=3.65$; $95 \%$ CI: 1.79-7.43) had higher odds of increasing consumption of fast-food meals. NonHispanic Blacks (OR = 1.45; 95\% CI: 1.02-2.06) and Hispanics (OR = 1.59; 95\% CI: 1.09-2.33) had higher odds of increasing alcohol drinking, while Asian Americans had lower odds of increasing alcohol drinking ( $\mathrm{OR}=0.51 ; 95 \% \mathrm{CI}$ : 0.35-0.74) and increasing smoking $(\mathrm{OR}=0.53 ; 95 \%$ CI: 0.29-0.97). Compared to individuals with high school education or less, those with Associate's degrees ( $\mathrm{OR}=1.46$; $95 \%$ CI: 1.03-2.08) or a bachelor's degree or higher ( $\mathrm{OR}=1.79 ; 95 \% \mathrm{CI}: 1.20-2.67)$ had higher odds of decreasing exercise time, and those with a bachelor's degree or higher had greater odds of increasing screen time $(\mathrm{OR}=1.90$; $95 \%$ CI: 1.27-2.85) and alcohol drinking (OR $=1.78 ; 95 \%$ CI: 1.11-2.87). Individuals who never married had higher odds of decreasing exercise time (OR $=1.43 ; 95 \% \mathrm{CI}: 1.02-2.01$ ) than married individuals. Individuals with household income between $\$ 25,000$ and $\$ 49,999$ $(\mathrm{OR}=0.37 ; 95 \%$ CI: $0.21-0.67)$ and $>\$ 50,000(\mathrm{OR}=0.22 ; 95 \%$ CI: $0.11-0.43)$ had lower odds of increasing cigarette smoking during the pandemic compared to those who had annual household income $<\$ 25,000$. Compared to individuals with private health insurance, those with Medicare had higher odds of decreasing exercise time (OR $=1.63 ; 95 \% \mathrm{CI}: 1.06-2.52$ ) and those with Medicaid had higher odds of increasing cigarette smoking (OR $=2.30 ; 95 \%$ CI: 1.03-5.17). 
Table 3. Multivariable associations between sociodemographic variables and the undesired changes of lifestyle before and during the COVID-19 pandemic.

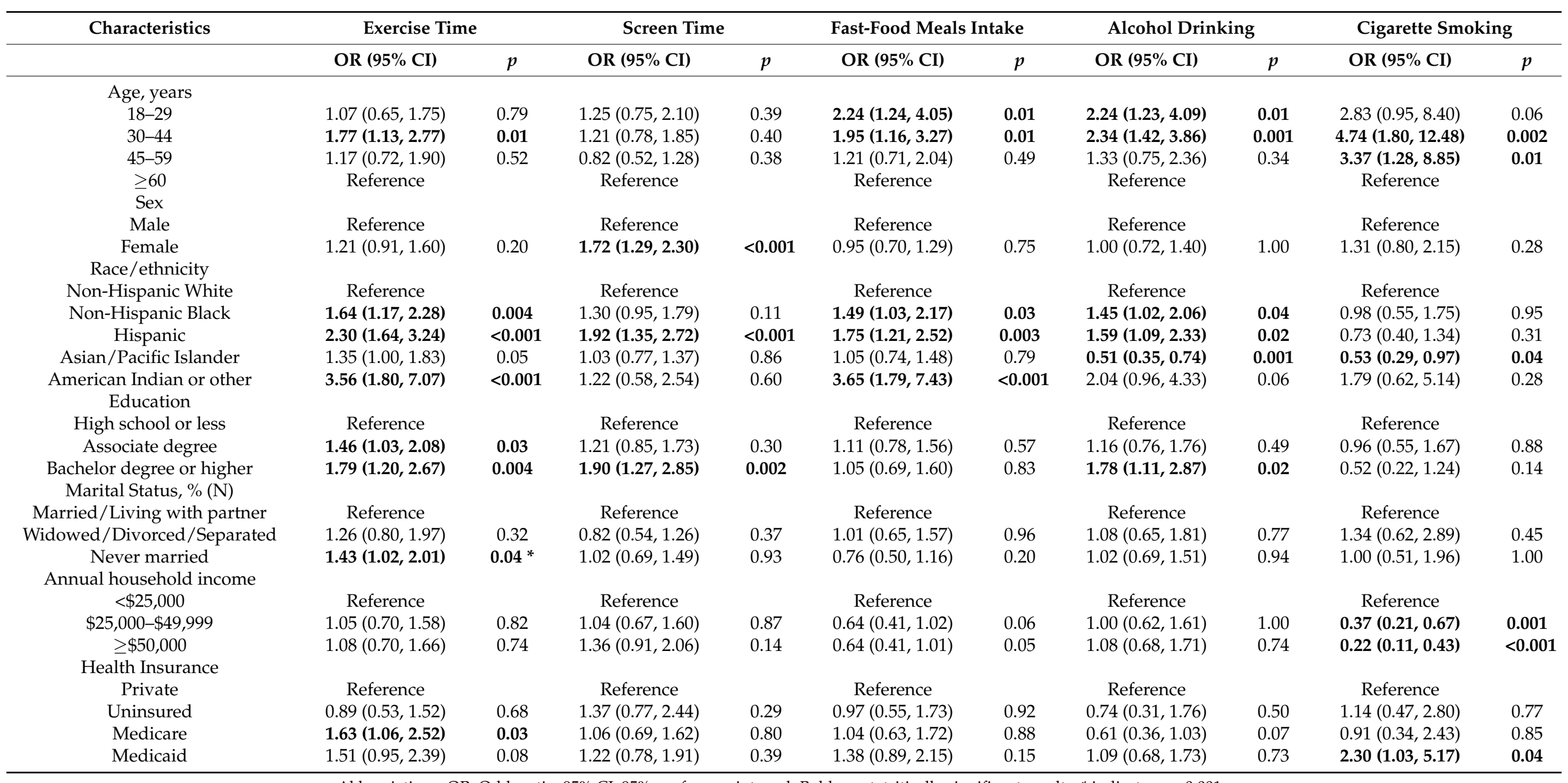

Abbreviations: OR: Odds ratio; $95 \%$ CI: $95 \%$ conference interval. Bold are statsitically significant results. * indicates $p<0.001$. 


\section{Discussion}

In this large study among USA adults with multiple racial/ethnic groups, we found that the COVID-19 pandemic resulted in undesired changes in multiple health-related lifestyle behaviors. On average, USA adults decreased their time spent on exercises, but increased their screen time during the COVID-19 pandemic compared to before. The percentage of heavy alcohol drinkers and cigarette smokers also increased during the pandemic. Our findings corroborate results from several recent studies conducted in other countries. We also identified subgroups who were more vulnerable to unfavorable lifestyle changes during the pandemic. The most critical findings are for age and racial groups. Compared to Non-Hispanic Whites, Non-Hispanic Blacks and Hispanics were more likely to have undesired changes in multiple lifestyle behaviors, including exercise, screen time, fast food intake, and alcohol drinking; American Indians and those in the "other" racial category were more likely to decrease their exercise time and increase consumption of fast-food meals; while Asian Americans were less likely to increase alcohol drinking and cigarette smoking. The clustering of negative lifestyle changes among racial and ethnic minority groups, as revealed in this study, is consistent with findings from previous studies documenting the disproportionate exposure to and suffering from the COVID-19 pandemic by these groups [17-20]. Younger-aged adults were more likely to have undesired changes in exercise time, consumption of fast-food meals, alcohol drinking, and cigarette smoking compared with old adults. In addition, females had higher odds to increase screen time during the pandemic than otherwise similar males; married individuals were not more likely to decrease exercise time during the pandemic than those who were married or lived with partners; higher household income was associated with lower the odds of smoking during the pandemic; individuals with higher education levels had higher odds of having more undesired lifestyle changes during the pandemic, including less exercise time, more screen time, and more alcohol drinking Future lifestyle interventions (e.g., promoting more exercise, reducing screening time, reducing smoking, and alcohol drinking counseling) might become more effective if they can target these high-risk subgroups who are more likely to be disproportionately impacted and have more undesired lifestyle changes in a future pandemic or potential new COVID-19 outbreak.

It is no surprise that the pandemic resulted in a decline in exercise time and a rise in screen time. Indeed, $20.9 \%$ of individuals did not do any exercise during the pandemic (vs. $15.3 \%$ before) and $79.8 \%$ of individuals had screen time $\geq 4 \mathrm{~h}$ per day (vs. $59.5 \%$ before). The proportion of individuals who exercised $\geq 30 \mathrm{~min}$ per day also decreased to $45.4 \%$ during the pandemic (vs. $56.4 \%$ before). On average, the daily time spent on exercise decreased from $38.65 \mathrm{~min}$ in pre-pandemic to $32.06 \mathrm{~min}$ during the pandemic and screen time increased from 5.06 to $6.79 \mathrm{~h}$. These findings are consistent with studies from other countries and populations [7-9,12]. For example, results from an international online survey showed that the COVID-19 had negative impact on all physical activity intensity levels (e.g., vigorous, moderate, walking) and the daily sitting time increased from 5.31 to $8.41 \mathrm{~h} \mathrm{[12].} \mathrm{In} \mathrm{a} \mathrm{study} \mathrm{conducted} \mathrm{in} \mathrm{Italy,} 56 \%$ of the study participants reported a reduced time for physical activity [8], where in our study 31.2\% Americans reported a decrease in exercise time during the pandemic. Such changes toward a more sedentary lifestyle are likely caused by the restrictions of outdoor activities, in-gym exercise, and working from home. A study conducted in Italy found that the frequencies of running, walking, swimming, and in-gym training were all reduced during the COVID-pandemic compared to before the pandemic [9]. Given the health benefits of exercise and the harm of screen time (as an indicator of a sedentary lifestyle), such undesired changes are likely to lead to adverse influences on various health outcomes. Exercise and screen time are independently linked to multiple health outcomes in almost all age groups. Although the observed changes are small to modest, the cumulative impact of such negative changes over several months or longer could have extensive consequences.

We found that the COVID-19 pandemic had a negative impact on alcohol drinking and cigarette smoking among USA adults, which is in line with findings from studies conducted 
in the USA $[14,15]$ and other countries such as Italy and Poland $[8,11]$. In our study, $9.0 \%$ of participants reported an increase in smoking during the pandemic, whereas this number was 29.5 and $45.2 \%$ in Italy and Poland, respectively $[8,11]$. Among smokers, the percentage of heavy smokers (i.e., $\geq 11$ cigarettes/day) also increased to $7.9 \%$ during the pandemic (vs. $5.8 \%$ before). In our study population, $23.2 \%$ of participants had increased alcohol drinking and the percentage of heavy drinkers (i.e., $\geq 5$ times/week) increased from $20.9 \%$ before the pandemic to $25.7 \%$ during the pandemic. Both alcohol drinking and smoking are risk factors for many health outcomes and have deleterious effects on the immune system, and therefore, can potentially increase the risk of SARS-CoV-2 infection and severity of the disease [21]. In addition, the evidence suggests that smoking is associated with a greater risk of adverse COVID-19 outcomes. Current and former smokers are 2.4 times more likely to need intensive care unit (ICU) support or die, compared with non-smokers [22]. The reasons for these negative changes are likely due to the stress and boredom of home confinement, as well as general life difficulties exacerbated by the COVID-19 pandemic [14]. Further studies are needed to understand the underlying causes and identify effective interventions in such emergency situations.

The observed decrease in fast food consumption is likely due to the stay-at-home order and the closure of fast-food restaurants during the pandemic. The average level of fast-meal consumption decreased from 1.41 times/week before the pandemic to 0.96 times/week during the pandemic. The percentage of individuals who reported consuming fast-food meals $\geq 3$ times /week also decreased from $37.7 \%$ before the pandemic to $33.3 \%$ during the pandemic. Although the majority of study participants (77.4\%) decreased or did not see a change in their fast-food meal consumption, there were still $22.6 \%$ that increased their fast-food meal consumption during the pandemic. In previous studies assessing different perspectives on eating, people reported more unhealthy dietary behaviors during the pandemic, including increased consumption of unhealthy foods (e.g., sweets), snacks, and the overall number of main meals $[8,11,12]$. Nevertheless, these data suggest that the COVID-19 pandemic and related regulations dramatically influence people's eating behaviors and food consumption patterns. Although many health organizations have provided guidelines for healthy eating during the COVID-19 pandemic and disseminated information on how adequate nutrition can support the immune system [23,24], people from multiple countries did not put these recommendations into practice during the pandemic.

The strengths of this study include a large, nationally representative sample, which is based on a population of USA adults with adequate numbers from each racial/ethnic group, and the timing of the data collection. Our study sample included over 1000 Asian Americans, who are commonly underrepresented or excluded in other studies. The study survey was designed and carried out in fall 2020 at the peak of the USA COVID-19 pandemic; thus, the survey responses are timely and likely to reflect the acute impacts of the pandemic. This study has several noteworthy limitations. First, the survey data were only collected at the one-time point during the pandemic. Therefore, the changes in lifestyle behaviors may only reflect the peak of the pandemic. Second, we were only able to collect limited information for each lifestyle behavior due to the concerns regarding participant time and burden. For dietary intake, we only focused on the consumption of fast-food meals.

\section{Conclusions}

In this large study conducted during the peak of the COVID-19 pandemic in the USA, we found that regulations to restrict non-essential activities and the stay-at-home order during the pandemic had profound impacts on multiple lifestyle behaviors in American adults. We found a marked increase in sedentary behaviors, alcohol consumption, and cigarette smoking and a decline in exercise. These negative changes in lifestyle disproportionately impact racial and ethnic minorities who also bear a higher disease burden of COVID-19. Our findings corroborate results from several recent studies conducted in 
other countries. Collectively, the observed undesired lifestyle changes may have a negative impact on both physical and mental health outcomes. Future studies investigating whether such lifestyle changes persist as the pandemic continues and whether the quality of life and health well-being are subsequently affected, are warranted. Resources and supports that can help people maintaining healthy lifestyles during the pandemic are urgently needed, and different strategies may be needed for different sub-groups.

Author Contributions: Fundraising: D.S.; Survey Design: L.C., L.S., D.Z., Z.C., X.H., Y.L., H.L., M.W., D.S.; Conceptualization: L.C., J.L., T.-S.T.; Data analysis: T.X., T.A.M.; Writing-original draft: L.C., J.L.; Writing-review and editing: L.C., J.L., T.X., T.A.M., T.-S.T., L.S., D.Z., Z.C., X.H., Y.L., H.L., M.W., D.S. All authors have read and agreed to the published version of the manuscript.

Funding: The "Health, Ethnicity, and Pandemic" (HEAP) study was funded by the Center for Reducing Health Disparities at University of Nebraska Medical Center, The Chinese Economists Society, and Calvin J. Li Memorial Foundation. Tong Xia was supported by a scholarship from Department of Epidemiology at the University of California Los Angeles (UCLA).

Institutional Review Board Statement: The study was approved by the Institutional Review Board at the National Opinion Research Center (NORC), University of Chicago.

Informed Consent Statement: Informed consent was obtained from all subjects involved in the study.

Data Availability Statement: The data presented in this study are available on request from the corresponding author and the Center for Reducing Health Disparities at University of Nebraska Medical Center.

Acknowledgments: We thank the "Health, Ethnicity, and Pandemic" (HEAP) study participants for their time and effort to fill the study survey. We also thank the National Opinion Research Center (NORC) for survey administration and data collection L. Chen has full access to all the data in the study and takes responsibility for the integrity of the data and the accuracy of the data analysis. All authors state no conflicts of interest.

Conflicts of Interest: The authors declare no conflict of interest. The funders had no role in the design, execution, interpretation, or writing of the study.

\section{References}

1. Piret, J.; Boivin, G. Pandemics Throughout History. Front. Microbiol. 2020, 11, 631736. [CrossRef] [PubMed]

2. Wiersinga, W.J.; Prescott, H.C. What Is COVID-19? JAMA 2020, 324, 816. [CrossRef] [PubMed]

3. Wiersinga, W.J.; Rhodes, A.; Cheng, A.C.; Peacock, S.J.; Prescott, H.C. Pathophysiology, Transmission, Diagnosis, and Treatment of Coronavirus Disease 2019 (COVID-19): A Review. JAMA 2020, 324, 782-793. [CrossRef] [PubMed]

4. WHO. WHO COVID-19 Dashboard. Available online: https://covid19.who.int (accessed on 6 September 2021).

5. Lau, J.T.; Yang, X.; Tsui, H.; Pang, E.; Kim, J.H. SARS preventive and risk behaviours of Hong Kong air travellers. Epidemiol. Infect. 2004, 132, 727-736. [CrossRef] [PubMed]

6. Tan, X.; Li, S.; Wang, C.; Chen, X.; Wu, X. Severe acute respiratory syndrome epidemic and change of people's health behavior in China. Health Educ. Res. 2004, 19, 576-580. [CrossRef] [PubMed]

7. Moore, S.A.; Faulkner, G.; Rhodes, R.E.; Brussoni, M.; Chulak-Bozzer, T.; Ferguson, L.J.; Mitra, R.; O’Reilly, N.; Spence, J.C.; Vanderloo, L.M.; et al. Impact of the COVID-19 virus outbreak on movement and play behaviours of Canadian children and youth: A national survey. Int. J. Behav. Nutr. Phys. Act. 2020, 17, 85. [CrossRef] [PubMed]

8. Ferrante, G.; Camussi, E.; Piccinelli, C.; Senore, C.; Armaroli, P.; Ortale, A.; Garena, F.; Giordano, L. Did social isolation during the SARS-CoV-2 epidemic have an impact on the lifestyles of citizens? Epidemiol. Prev. 2020, 44 (Suppl. 2), 353-362. [PubMed]

9. Di Renzo, L.; Gualtieri, P.; Pivari, F.; Soldati, L.; Attina, A.; Cinelli, G.; Leggeri, C.; Caparello, G.; Barrea, L.; Scerbo, F.; et al. Eating habits and lifestyle changes during COVID-19 lockdown: An Italian survey. J. Transl. Med. 2020, 18, 229. [CrossRef] [PubMed]

10. Werneck, A.O.; Silva, D.R.; Malta, D.C.; Gomes, C.S.; Souza-Junior, P.R.; Azevedo, L.O.; Barros, M.B.; Szwarcwald, C.L. Associations of sedentary behaviours and incidence of unhealthy diet during the COVID-19 quarantine in Brazil. Public Health Nutr. 2021, 24, 422-426. [CrossRef] [PubMed]

11. Sidor, A.; Rzymski, P. Dietary Choices and Habits during COVID-19 Lockdown: Experience from Poland. Nutrients 2020, $12,1657$. [CrossRef] [PubMed]

12. Ammar, A.; Brach, M.; Trabelsi, K.; Chtourou, H.; Boukhris, O.; Masmoudi, L.; Bouaziz, B.; Bentlage, E.; How, D.; Ahmed, M.; et al. Effects of COVID-19 Home Confinement on Eating Behaviour and Physical Activity: Results of the ECLB-COVID19 International Online Survey. Nutrients 2020, 12, 1583. [CrossRef] [PubMed] 
13. Barone Gibbs, B.; Kline, C.E.; Huber, K.A.; Paley, J.L.; Perera, S. Covid-19 shelter-at-home and work, lifestyle and well-being in desk workers. Occup. Med. 2021, 71, 86-94. [CrossRef] [PubMed]

14. Grossman, E.R.; Benjamin-Neelon, S.E.; Sonnenschein, S. Alcohol Consumption during the COVID-19 Pandemic: A CrossSectional Survey of US Adults. Int. J. Environ. Res. Public Health 2020, 17, 9189. [CrossRef] [PubMed]

15. Pollard, M.S.; Tucker, J.S.; Green, H.D., Jr. Changes in Adult Alcohol Use and Consequences During the COVID-19 Pandemic in the US. JAMA Netw. Open 2020, 3, e2022942. [CrossRef] [PubMed]

16. Montgomery R, D.J.; Ganesh, N. Response rate Calculation Methodology for Recruitment of a Two-Phase Probability-Based Panel: The Case of AmeriSpeak; University of Chicago National Opinion Research Center white Pape. 2016. Available online: https: / / www.google.com / url?sa=t\&rct=j\&q=\&esrc=s\&source=web\&cd=\&cad=rja\&uact=8\&ved=2ahUKEwiEwt7B25jzAhX SLH0KHRZKBBQQFnoECAIQAQ\&url=https\%3A\%2F\%2Famerispeak.norc.org\%2FDocuments\%2FResearch\%2FNORC_Labs _and_Amerispeak_launch_plans_Nov_2016_DTP_Formatted.pdf\&usg=AOvVaw2upuCUtFKREntT7zZI83n9 (accessed on 16 March 2021).

17. Karaca-Mandic, P.; Georgiou, A.; Sen, S. Assessment of COVID-19 Hospitalizations by Race/Ethnicity in 12 States. JAMA Intern. Med. 2021, 181, 131-134. [CrossRef] [PubMed]

18. Laurencin, C.T.; McClinton, A. The COVID-19 Pandemic: A Call to Action to Identify and Address Racial and Ethnic Disparities. Morb. Mortal. Wkly. Rep. 2020, 7, 398-402. [CrossRef] [PubMed]

19. Moore, J.T.; Ricaldi, J.N.; Rose, C.E.; Fuld, J.; Parise, M.; Kang, G.J.; Driscoll, A.K.; Norris, T.; Wilson, N.; Rainisch, G.; et al. Disparities in Incidence of COVID-19 Among Underrepresented Racial/Ethnic Groups in Counties Identified as Hotspots During June 5-18, 2020-22 States, February-June 2020. Morb. Mortal. Wkly. Rep. 2020, 69, 1122-1126.

20. Tai, D.B.G.; Shah, A.; Doubeni, C.A.; Sia, I.G.; Wieland, M.L. The Disproportionate Impact of COVID-19 on Racial and Ethnic Minorities in the United States. Clin. Infect. Dis. 2021, 72, 703-706. [CrossRef] [PubMed]

21. Grundy, E.J.; Suddek, T.; Filippidis, F.T.; Majeed, A.; Coronini-Cronberg, S. Smoking, SARS-CoV-2 and COVID-19: A review of reviews considering implications for public health policy and practice. Tob. Induc. Dis. 2020, 18, 58. [CrossRef] [PubMed]

22. Vardavas, C.I.; Nikitara, K. COVID-19 and smoking: A systematic review of the evidence. Tob. Induc. Dis. 2020, 18, 20. [CrossRef] [PubMed]

23. Blog, H.H. Available online: https:/ / www.health.harvard.edu/blog/eating-during-covid-19-improve-your-mood-and-lower-s tress-2020040719409 (accessed on 13 September 2021).

24. Academy of Nutrition and Dietetics. Eating well during Coronavirus/COVID-19. Available online: https://www.eatright.org/c oronavirus (accessed on 13 September 2021). 